\title{
DISEÑO DE MODELOS DE PROCESOS PRODUCTI- VOS EN INGENIERÍA POR SIMULACIÓN
}

\section{Carlos Sánchez Guzmán}

\section{Resumen}

En el diseño de procesos se utilizaron probabilidades para estimar la transición de un estado a otro. Es un método de validar una hipótesis, por distribución estadística.

Los diseños de experimentos aplicados, permitieron modelar y evaluar diversos problemas a través de técnicas estadísticas, como el análisis de varianza para contrastar hipótesis con conceptos de Fisher.

En el modelo, se formuló y estableció sus principales parámetros. Los modelos clásicos son los sistemas económicos y empresariales, sin embargo, la metodología se puede adaptar muy fácilmente a sistemas sociales, políticos, psicológicos, económicos o de ingeniería.

Palabras clave: simulación, diseño, números aleatorios.

\section{Abstract}

In the design process probabilities are used to estimate the transition from one state to another. It is a method to validate a hypothesis, by statistical distribution. The design of experiments applied, allowed to model and evaluates various problems through statistical techniques, such as analysis of variance to test hypotheses with concepts of Fisher.

In the model, was formulated and established its main parameters. Classic models are economic and business systems, however, the methodology can be easily adapted to social, political, psychological, economic or engineering systems.

Keywords: Simulation, design, random numbers. 


\section{INTRODUCCIÓN}

La simulación hace uso de muchas técnicas analíticas y de computadoras. Para analizar sistemas económicos, la simulación se convierte en un medio esencial. Observar el comportamiento real de un sistema, con frecuencia es extremadamente costoso o imposible cuando se trata de estudiar la etapa de observación del método científico. Podría ocurrir que no se cuente con datos históricos de algunas variables. Sin embargo, se puede contar con la suficiente información para formular una hipótesis respecto a las distribuciones de probabilidad de algunas de las variables con respecto al tiempo, o sobre los cálculos de sus tendencias con respecto al tiempo. En este caso, se generan datos con la computadora para un sistema, basadas en distribuciones de probabilidad supuestas o en término a sus tendencias. Por medio de las observaciones, se puede formular, manipular y probar modelos que describan el comportamiento del sistema como un todo.

El punto de partida de cualquier experimento de simulación en computadora es un modelo del sistema simulado. En otras palabras, se supone que ya se ha formulado el modelo y se han especificado sus parámetros. Regularmente, se evalúan modelos económicos y empresariales, sin embargo, la metodología se puede adaptar a sistemas de otras áreas del saber humano.

Entre algunos de los modelos para proceder a simular, tenemos a las cadenas de Markov, colas, inventarios,

mercado, producción, financieros, incorporados y juegos de gerencia. De igual manera, modelos de empresas e industrias. A veces, una compañía puede experimentar con diferentes normas de publicidad y mercadotecnia y comparar los resultados obtenidos. Sin embargo, aún en el corto número de casos en que se pueden efectuar experimentos reales con sistemas económicos, no es muy probable que se puedan mantener constantes todas las variables, a fin de obtener comparaciones efectivas de las diversas normas económicas.

Cuando es imposible o poco práctico efectuar una experimentación controlada y se carece de datos representativos, o estos contienen un error aleatorio excesivo, se diseña el modelo y se calcula sus parámetros del sistema que relacione las variables endógenas (dependientes) con las variables exógenas (independientes). Si en algún caso, el modelo toma la forma de un número relativamente pequeño de ecuaciones de primer o segundo grado simultáneas, diferenciales lineales o de diferenciación, se pueden emplear técnicas analíticas para evaluar los efectos de los casos alternativos. Por otro lado, si el modelo se compone de un gran número de ecuaciones simultáneas de grados superiores diferenciales no lineales o de diferenciación, que incluyen términos de error estocástico, entonces las técnicas analíticas pueden existir solo en teoría. En tal caso, es necesario recurrir a los análisis numéricos o simulación para evaluar todas las posibilidades. 


\section{IDENTIFICACIÓN DEL PROBLEMA}

En casi todas las actividades humanas existen problemas de naturaleza compleja, en las que no existen técnicas apropiadas para resolverlos. Los modelos usados en el desarrollo de teorias de ciclos de negocios y el comportamiento de mercado, dan origen a dificultades de esta índole. Desde mucho tiempo atrás, se ha confiado en soluciones de ecuaciones diferenciales y de diferenciación como técnicas analíticas estándar para investigar el comportamiento de ciclos de negocios.

Los sistemas complejos no presentan un comportamiento que pueda expresarse matemáticamente de manera sencilla. Si los sistemas se componen de ecuaciones de diferenciación no lineal o diferenciales, caracterizados por estructuras complejas de demora y por la inclusión de variables aleatorias, entonces es posible encontrar soluciones analíticas. En muchos problemas de las ciencias económicas y administrativas, la simulación puede ser el único instrumento de que dispone el analista. Más aún, en algunos casos, a pesar de que se sospeche que existe una solución analitica (aunque no se esté familiarizado con ella), utilizar la simulación puede ser menos costosa en función del tiempo del analista y de la computadora. En otras palabras, la información adicional obtenida mediante una solución analítica (en caso de que exista) puede no ser suficiente para justificar el tiempo de investigación de la técnica analítica y el tiempo requerido para su ejecución.
La simulación es útil cuando se dificulta o imposibilita la solución del modelo analítico o numérico requerido en un determinado problema. Por tal motivo, planteamos el problema: ¿los diseños de experimentos de simulación proporcionan resultados óptimos en ingeniería?

\section{MARCO TEÓRICO}

La idea de simulación abarca conceptos básicos, como lo siguiente. Sea Y el símbolo de una variable de salida (endógena) de un sistema que se desea estudiar, X el simbolo de las variables $\mathrm{k}$ (exógenas y normativas) que se piensa influye en Y, de acuerdo a la relación funcional:

$$
\mathrm{Y}=\varnothing(\mathrm{x})
$$

En los textos sobre diseño experimental, se dice que $\mathrm{Y}$ es una respuesta y que los valores de Xi ( $\mathrm{i}=1,2, \ldots, \mathrm{k})$, son los factores. La función $\varnothing$ se denomina superficie de respuesta. Un caso especial es el modelo lineal simple:

$$
\mathrm{y}=\sum_{1} \theta i X i
$$

en donde los valores $\theta \mathrm{i}$ son los parámetros. Si fuera posible llevar a cabo la experimentación, se podría variar X, observar Y, determinar los valores de los parámetros $\theta$, y luego interpretar el modelo adaptado:

$$
\hat{\mathrm{Y}}=\sum{ }^{\wedge} \mathrm{i} \mathrm{Xi}
$$

en donde ${ }^{\wedge} i, \hat{Y}$ indican los valores calculados $\theta$ ’i, Y, respectivamente. 
Un autor clásico, Prawda (1993), describe al lenguaje de simulación GPSS mostrando las ventajas de utilizar lenguajes especiales. De igual manera, ilustra la simulación de procesos continuos a través de la dinámica industrial, aportando conceptos asociados a los juegos de simulación, herramientas dinámicas de aprendizaje para la toma de decisiones, mostrando procesos de producción como de gestión corporativa; de igual manera, la verificación estadística de los resultados de un modelo de simulación. Asimismo, define que la simulación es útil cuando se dificulta o imposibilita la resolución del modelo analítico o numérico requerido en un determinado problema. Comparados con los modelos analíticos y numéricos, los procesos de simulación presentan ventajas y desventajas. Por un lado, permiten estudiar el sistema real sin deformarlo. Los modelos analíticos o numéricos requieren la simplificación del sistema real de estudio, a fin de que se apegue a las condiciones que fundamentan la teoría del modelo en uso; por esto, finalmente muchos modelos analíticos y numéricos resuelven un sistema deformado muy lejano al sistema real bajo estudio.

Naylor (1982), a efectos de hacer más realista el modelo, agrega una variable fortuita, expresando el modelo,

$$
\hat{\mathrm{Y}}=\sum_{1} i X i
$$

en donde la función densidad de probabilidad de $\in$ está dada por $\mathrm{f}(\mathcal{C}$, Y), y representa los parámetros de la distribución. Para hacer el modelo más real, se pueden incluir en el modelo las transformaciones de $\mathrm{g}$ (y) y $\mathrm{h}$ (xi), que aparecen en la respuesta, o en uno o más valores de x. Alguna de estas transformaciones pueden implicar no linealidades, al igual que parámetros adicionales. También se pueden introducir otras variables fortuitas, que se incluyen con sus propios pesos como $\beta \mathrm{j}$, así como su distribución y parámetro. También se puede emplear una dependencia del tiempo, que se indica mediante el subíndice t. en general; el modelo es:

\section{$\mathbf{g}(\mathbf{y i})=, \mathbf{h}(\mathbf{x i}, \mathbf{t})+\mathbf{j} \psi(\mathbf{Z j t y j})+\mathbf{f}(\mathbf{e}, \mathbf{y})$}

Se pueden incluir variables ficticias que constan de unos y ceros, con los cuales se puede saber si existen o no ciertas variables en momentos determinados, e identifican bloques de variables que se usan juntas.

Moskowitz y Writght (1979), proporcionan razones que legitiman la simulación en computadoras como medio de análisis para modelos de sistemas de negocios y económicos, en la que existe similitud entre la simulación en computadora y las técnicas analíticas normales, como el cálculo diferencial, la programación matemática y el cálculo de variaciones.

En general, la razón principal para usar cualquiera de estos medios de análisis es la búsqueda de conocimientos científicos relacionados con el comportamiento de un sistema económico dado. Cuando se aplica a sistemas económicos, el método científico sigue el procedimiento conocido de cuatro etapas: 1) la observación 
de sistemas, 2) la formulación de un modelo matemático que trate de explicar las observaciones del sistema, 3) la predicción del comportamiento del sistema tomando como base el modelo, utilizando deducciones matemáticas o lógicas, es decir, mediante la obtención de soluciones al modelo, y 4) la realización de experimentos para comprobar la validez de dicho modelo.

Dominguez (1995), en "Dirección de Operaciones", explica los modelos de cadenas de Markov, que se describe mediante un número finito de estados diferentes. En cualquier instante dado en el tiempo, el proceso estará en uno de los estados diferentes. La probabilidad de que el proceso se encuentre en el estado $\mathrm{j}(\mathrm{j}=1,2, \ldots, \mathrm{M})$, al concluir el periodo dado, depende solo del estado del proceso $\mathrm{i}(\mathrm{i}=1,2, \ldots, \mathrm{M})$ al final del periodo anterior. Tal proceso se puede describir por completo mediante una matríz de transición $\mathrm{P}$, cuyos elementos pij denotan la probabilidad de pasar del estado i al estado j:

$$
\begin{gathered}
\mathbf{P}=[\mathbf{p 1 1} \mathbf{p} 12 \ldots \mathbf{p} 1 \mathbf{M} ; \mathbf{p 2 1}, \mathbf{p 2 2}, \ldots \\
\mathbf{p 2 M} ; \ldots \mathbf{p M 1} \mathbf{p M 2} \ldots \mathbf{p} \mathbf{M M}] \\
\text { en donde: } \\
\sum_{1} p_{i j}=1, \mathrm{i}=1,2 \ldots \mathrm{M}
\end{gathered}
$$

Los modelos de cadena de Markov se han usado a menudo en la mercadotecnia, para simular la preferencia de marcas de los consumidores. En este caso, los estados corresponden a diferentes preferencias de marcas para un artículo en particular.

Forrester desarrolló su propio conjunto de símbolos para el diagrama de flujo y el lenguaje de simulación para objetivos especiales, DYNAMO, a fin de simular el comportamiento de sistemas económicos y de negocios que se puedan describir mediante un grupo de ecuaciones de diferencias repetitivas.

Para verificar que los números pseudoaleatorios tienen una distribución uniforme en el intervalo cerrado $[0,1]$, se sigue la siguiente secuenci: 1) se formula la hipótesis Ho, de que los números provienen de una distribución uniforme, 2) se selecciona una muestra de tamaño $\mathrm{n}$ de números pseudoaleatorios generados, 3) se calcula la función de distribución acumulada empírica, 4)se evalua la estadística de $\mathrm{k}$ - v, a partir de:

$$
\mathbf{D}=\operatorname{máx}[\mathbf{F n}(\mathbf{x i})-\mathrm{xi}], \mathbf{0} \leq \mathrm{x}_{\mathrm{i}} \leq \mathbf{1}
$$

Consultar la tabla de límites con la prueba k - v. Si D es menor o igual a este número, se acepta Ho; de otra manera, se rechaza Ho.

Al calcular números aleatorios, se tiene:
0.10
0.32
0.76
0.13
0.34
0.37
0.04
0.64
0.74
0.24
etc etc etc etc etc

Si se desea probar la hipótesis Ho: provienen de una distribución uniforme en $[0,1]$, a un nivel de significancia del $90 \%$.

Se arregla la tabla de tal manera que, $\mathrm{x}_{\mathrm{i}} \leq \mathrm{x}_{\mathrm{i}+1}$

1. 0.02

2. 0.02

3. 0.03

4. 0.04

etc 
Se construye Fn $\left(\mathrm{x}_{\mathrm{i}}\right)$,

$$
\begin{gathered}
\text { Fn }(0.00)=0.00 \\
\text { Fn }(0.02)=0.04 \\
\text { Fn }(0.03)=0.06 \\
\text { Fn }(0.04)=0.08 \\
\text { etc }
\end{gathered}
$$

Luego determinamos

$\mathrm{D}=\operatorname{máx}\left[\mathrm{Fn}\left(\mathrm{x}_{\mathrm{i}}\right)-\mathrm{x}_{\mathrm{i}}\right]=0.12$

y ocurre cuando Fn (0.38)

Por tanto, para un nivel de significancia del $90 \%$ y una muestra de 50 números, si tiene el valor tabulado de 0.172. Como D < 0.172, se acepta Ho: los 50 números sí provienen de una distribución uniforme en el intervalo cerrado $[0,1]$.

Cuando es posible encontrar la función inversa $\mathrm{F}^{-1}(\mathrm{r})=\mathrm{x}$, se pueden generar variables aleatorias con la distribución $F(\bullet)$, a partir de variables aleatorias $r$, distribuidas uniformemente en el intervalo cerrado $[0,1]$. Este proceso se conoce como transformación inversa. Se conoce $\mathrm{F}(\mathrm{x})$, que es la distribución deseada y se genera un número aleatorio de una distribución uniforme en el intervalo [0, 1]. Entonces, el número aleatorio con distribución $\mathrm{F}(\mathrm{x})$ se calcula de la función inversa.

Se generan números aleatorios distribuidos exponencialmente con media 1/0. Se tiene:

$$
\mathbf{r}=\mathbf{F}(\mathbf{t})=\mathbf{1}-\exp (-\boldsymbol{\theta} \mathbf{t})
$$

por lo que la transformación inversa procede de la siguiente manera:

$\mathbf{1}-\mathbf{r}=\exp (-\theta \mathbf{t})$

$\log (1-\mathbf{r})=-\theta \mathbf{t}$

$\mathbf{t}=-\log (1-\mathbf{r}) \theta$
Se genera un número aleatorio $r$, distribuido uniformemente en el intervalo $[0,1]$.

Montgomery (2000), explica la distribución exponencial, donde $\mathrm{t}$ es una variable aleatoria distribuida exponencialmente, con media $1 / \theta$, con función densidad $\mathrm{f}(\mathrm{t})=\theta \exp (-\theta \mathrm{t})$, con distribución $F(t)=1-\exp (-\theta t)$, entonces $\mathrm{t}=-\log (1-\mathrm{r}) / \theta$, donde $\mathrm{r}$ es una variable aleatoria con distribución uniforme en [0, 1].

En cuanto a la distribución de Erlang, se tiene $\mathrm{t}$ como variable aleatoria, media $\mathrm{k} / \lambda$, varianza $\mathrm{k} / \lambda^{2}$, $\mathrm{y}$ la densidad correspondiente. La función inversa es $t=F^{-1}\left(r_{1} . r_{2}, \ldots, r_{k}\right)$, siendo $r_{1}, r_{2}, \ldots, r_{k}$ variables aleatorias independientes con distribución uniforme en $[0,1]$.

De igual manera, se presenta la distribución normal, distribución ji - cuadrada, distribución de Poisson, distribución log normal, distribución geométrica, distribución binomial negativa, distribución binomial, distribuciones empíricas, siendo las principales distribuciones que generan números aleatorios.

Hillier (2001), en cuanto a los modelos de simulación, afirma que en eventos discretos se utilizan para estudiar las características de operación de líneas de espera simples y complejas. Cuando se tienen distribuciones empíricas y estructuras complejas, los modelos analíticos no trabajan y se tienen que utilizar procesos de simulación. Los eventos discretos de la simulación describen cómo los "clientes" o "elementos" fluyen a través del 
sistema bajo estudio conforme pasa el tiempo. Los modelos de simulación discretos suponen que los cambios en el sistema ocurren instantáneamente en puntos específicos de tiempo. Se define por evento al instante en que ocurren estos cambios.

La simulación se puede hacer manualmente o utilizando lenguajes de programación, como el GPSS, GASP, DYNAMO, Fortran, etc.

El enfoque del flujo de procesos consiste en desarrollar un diagrama de bloques, donde cada bloque describe una actividad relacionada con el "cliente", a medida que éste pasa por el sistema. A las actividades se les asocia una variable aleatoria relativa al tiempo de duración de la misma. E1 diagrama de bloques refleja las posibles bifurcaciones que el cliente puede, en un momento dado, elegir cuando ejerce una decisión. El lenguaje de simulación GPSS está basado en este enfoque.

Asimismo, el enfoque de programación de eventos se centra en dos características fundamentales:

a) Se deben describir los eventos individuales que cambian el estado del sistema en diferentes periodos de tiempo.

b) Se deben prever los cambios futuros que el sistema va a experimentar, a través de la creación de una "lista de eventos futuros".

El estado del sistema en cualquier instante de tiempo lo describen las condiciones o atributos de los elementos del mismo en ese momento. Por ejemplo, los atributos de cada carro que entra a la gasolinera serán: el tipo de gasolina, la capacidad de su tanque, etc.

\section{SIMULACIÓN DE UN PROCESO DISCRETO}

Se evalúa el comportamiento de una gasolinera con $\mathrm{n}$ locales, $\mathrm{m}$ bombas con 2 mangueras. La gasolinera vende 3 tipos de combustible: $\mathrm{N}, \mathrm{E}$, $\mathrm{D}$; por lo tanto, una determinada sede tiene $\mathrm{k}$ mangueras de gasolina $\mathrm{N}, \mathrm{j}$ de $\mathrm{E}, \mathrm{W}$ de $\mathrm{D}$.

El estudio previo muestra que la llegada de vehículos a esa gasolinera es una variable aleatoria con distribución de Poisson, con diferentes valores medios, en razón a la hora del día que se trate.

Se tiene, de 6 a las 7 a.m., el promedio es de 5 vehículos/hora; de 7 a 9, el promedio es 15 vehículos/hora; y 10 de las 9 a las 14 horas. Se procede a simular la operación de 6 a 14 horas de un día de trabajo. Se ha llegado a obtener la siguiente información:

a) $50 \%$ de los autos son chicos y tienen tanques de 40 litros.

b) $30 \%$ de los autos son medianos y tienen tanques de 60 litros.

c) $15 \%$ de los autos son grandes y tienen tanques de 80 litros.

d) $5 \%$ son camiones y tienen tanques de 150 litros.

El estudio de mercado revela que los autos chicos y medianos cargan $\mathrm{N}$ el $100 \%$ de las veces; los grandes, en un $60 \%$ de los casos es una mezcla; y el $40 \%$ es del tipo E. El $30 \%$ de los automovilistas requieren únicamente 
de un servicio de gasolina, el $70 \%$ de otros servicios. El estado del sistema se analiza cada 5 minutos. Los tiempos medios de servicio y su desviación estándar:

\begin{tabular}{|c|c|c|c|}
\hline Servicio & $\begin{array}{c}\text { Cada } \\
5 \text { min }\end{array}$ & $\begin{array}{c}\text { Media } \\
(\mathrm{min})\end{array}$ & $\begin{array}{c}\text { Desviación } \\
(\mathrm{min})\end{array}$ \\
\hline Carga & & & \\
40 litros & 1 & 5 & 1 \\
60 litros & 1 & 5 & 2 \\
80 litros & 2 & 10 & 3 \\
150 litros & 3 & 15 & 4 \\
\hline Otros & & & \\
Chico & 2 & 10 & 2 \\
Mediano & 3 & 15 & 3 \\
Grande & 3 & 15 & 4 \\
Camión & 4 & 20 & 5 \\
\hline
\end{tabular}

Cuando el servicio está ocupado, existe $10 \%$ de probabilidad que un automóvil no entre a la gasolinera. Las probabilidades relativas a vehículos que abandonan la línea de espera, es:

\begin{tabular}{|c|c|}
\hline $\begin{array}{c}\text { "n" vehículos en } \\
\text { espera }\end{array}$ & $\begin{array}{c}\text { Probabilidad de } \\
\text { abandonar espera }\end{array}$ \\
\hline 1 & 0.20 \\
\hline 2 & 0.30 \\
\hline 3 & 0.40 \\
\hline 4 & 0.50 \\
\hline 5 & 0.60 \\
\hline 6 & 0.70 \\
\hline
\end{tabular}

El $80 \%$ de los vehículos abandona la gasolinera si tiene que esperar 20 minutos o más. Los pasos de la simulación, son:

1. El tiempo a considerar es de 5 minutos, como inicio es 6 a.m., y el final 14 horas.

2. Los clientes llegan de manera progresiva.

3. Se calcula las probabilidades de llegada, con distribución de Poisson.
4. Utilizando números aleatorios, se simula los eventos de acuerdo al método de distribuciones empíricas.

5. Simulación del tipo de vehículo:

\begin{tabular}{|l|l|l|l|}
\hline Tipo & Probabilidad & Servicio & INDICE \\
\hline Chico & 0.50 & 40 & $00-49$ \\
\hline Mediano & 0.30 & 60 & $50-79$ \\
\hline Grande & 0.15 & 80 & $80-94$ \\
\hline Camión & $\mathbf{0 . 0 5}$ & $\mathbf{1 5 0}$ & $\mathbf{9 5 - 9 9}$ \\
\hline
\end{tabular}

6) Simulación del tipo de combustible:

\begin{tabular}{|l|l|c|}
\hline \multicolumn{1}{|c|}{ Tipo } & Combustible & $\begin{array}{c}\text { Número } \\
\text { Aleatorio }\end{array}$ \\
\hline Chico & N & - \\
\hline Mediano & N & - \\
\hline Grande & E $(40 \%)$ & $00-39$ \\
\hline Grande & M (60\%) & $40-99$ \\
\hline Camión & D & $\mathbf{9 5 - 9 9}$ \\
\hline
\end{tabular}

7. Simulación del tipo de servicio:

\begin{tabular}{|l|c|c|}
\hline \multicolumn{1}{|c|}{ Servicio } & $\begin{array}{c}\text { Probabili- } \\
\text { dad }\end{array}$ & $\begin{array}{c}\text { Número } \\
\text { Aleatorio }\end{array}$ \\
\hline Combustible & 0.30 & $00-29$ \\
\hline $\begin{array}{l}\text { Combustible y } \\
\text { otros servicios }\end{array}$ & $\mathbf{0 . 7 0}$ & $\mathbf{3 0 - 9 9}$ \\
\hline
\end{tabular}

8. Simulación del tiempo de servicio de combustible:

\begin{tabular}{|c|c|c|c|c|}
\hline & \multicolumn{2}{|c|}{$\begin{array}{c}\text { Combusti- } \\
\text { bles }\end{array}$} & \multicolumn{2}{l|}{ Otros servicios } \\
\hline $\begin{array}{c}\text { Capa- } \\
\text { cidad }\end{array}$ & $\begin{array}{c}\text { Me- } \\
\text { dia }\end{array}$ & D. st. & Media & D.st. \\
\hline 40 & 5 & 1 & 10 & 2 \\
\hline 60 & 5 & 2 & 15 & 3 \\
\hline 80 & 10 & 3 & 15 & 4 \\
\hline $\mathbf{1 5 0}$ & $\mathbf{1 5}$ & $\mathbf{4}$ & $\mathbf{2 0}$ & $\mathbf{5}$ \\
\hline
\end{tabular}


La simulación supone que la carga del combustible es por la capacidad total del tanque del automóvil. En realidad se debería tener una distribución de diferentes rangos de carga por tipo de vehículo.

9. Probabilidades que un vehículo no entre al servicio:

\begin{tabular}{|c|c|c|}
\hline $\begin{array}{c}\text { Ta- } \\
\text { maño }\end{array}$ & $\begin{array}{c}\text { Probabilidad de no } \\
\text { ingreso }\end{array}$ & N. A. \\
\hline 0 & 0.10 & $00-09$ \\
1 & 0.20 & $00-19$ \\
2 & 0.30 & $00-29$ \\
3 & 0.40 & $00-39$ \\
4 & 0.50 & $00-49$ \\
5 & 0.60 & $00-59$ \\
6 & 0.70 & $00-69$ \\
\hline
\end{tabular}

La probabilidad de no esperar más de 20 minutos es igual a 0.80 y los números aleatorios son $00-79$.

10. Se procede a simular:

\begin{tabular}{|c|c|c|}
\hline Caso & Sedes & Atención \\
\hline 1 & 1 & 2 \\
2 & 2 & 3 \\
3 & 2 & 4 \\
4 & 3 & 5 \\
5 & 3 & 6 \\
6 & 3 & 7 \\
\hline
\end{tabular}

Se simula de 6 a 14 horas en intervalos de 5 minutos. El número 23 a las 6 de la mañana en la llegada indica que no ha llegado un cliente (vehículo).

\section{SOBRE MODELOS DE SIMULACIÓN}

Al realizar experimentos de simulación en computadora con el modelo de un sistema dado, lo que se desea conocer son los efectos que los dife- rentes niveles de factores tienen en las variables endógenas o de respuesta. En otras palabras, un experimento de simulación en computadora consiste en una serie de corridas de computadora en las que se prueban empíricamente (utilizando los datos tomados de la simulación) los efectos de niveles alternativos de los factores sobre los valores de las variables de respuesta. Las relaciones funcionales que describen la interacción de las variables y los componentes del modelo de un sistema de negocios o económico, son de dos tipos: las identidades y las ecuaciones de comportamiento. Tanto unas como otras se emplean para generar el comportamiento del sistema. Las identidades pueden tomar la forma de definiciones o proposiciones tautológicas respecto a los componentes del modelo.

Una relación de comportamiento es una hipótesis, casi siempre una ecuación matemática, que relaciona a las variables endógenas y de estado del sistema con sus variables exógenas. Como ejemplo de las ecuaciones de sistemas económicos, están las funciones de consumo e inversión para una industria y las funciones de producción para una compañía. Las ecuaciones de comportamiento para procesos estocásticos toman la forma de funciones de densidad de probabilidad.

- modelos DE CADENAS DE MARKOV. Un proceso de Markov es aquel que se describe mediante un número finito de estados diferentes. En cualquier instante dado en el tiem- 
po, el proceso estará en uno de los $\mathrm{M}$ estados diferentes. La probabilidad de que el proceso se encuentre en el estado $\mathrm{j}$ ( $\mathrm{j}=$ $1,2, \ldots, \mathrm{M})$ al concluir el periodo dado, depende solo del estado del proceso i)i $=1,2, \ldots, \mathrm{M}$ ) al final del periodo anterior. Tal proceso se puede describir por completo mediante una matriz de transición.

- MOdelos DE COlAS. Muchos sistemas industriales se caracterizan por el arribo de algún tipo de unidad de entrada a una o más estaciones de servicio. Estas entradas pueden ser órdenes de ventas, de producción, descomposturas de máquinas, aeroplanos que llegan a un aeropuerto o automóviles que 1legan a una gasolinera.

Entre los modelos de colas, tenemos: el modelo de canal único y estación única, el modelo de estaciones múltiples con un solo canal y el modelo de canales múltiples y estaciones múltiples.

- MODElOS DE INVENTARIO. Lo que se conoce como sistemas de inventario, constituye una tercera clasificación general de los sistemas industriales para los que la simulación en computadora puede ser un instrumento analitico de gran utilidad. Puesto que se presentan modelos de computadora tomados de varias aplicaciones, tales modelos reflejan la realidad. Es de esperar que el sistema seleccionado para ilustrar este punto contenga una muestra representativa de algunos de los elementos más importantes que generalmente se encuentran en los sistemas de inventario.

- MOdelos DE PRODUCCIÓN. El término "planeación de producción", se ha utilizado para describir una gran variedad de problemas industriales relacionados con la toma de decisiones sobre la asignación de recursos de manufactura en cada instante durante el periodo dado de planeación.

- Modelos de MERCAdoteCNIA. Se enfocan hacia el comportamiento del consumidor $y$ a la demandade productos. Algunos modelos de este tipo son: el modelo de Pillsburg, el de Anheuser - Busch, el de Corning Glass.

- MOdelos FinANCIEROS. Las finanzas son otro campo para simulación. Entre los modelos, tenemos la simulación de Clarkson, para inversiones en fideicomiso, el modelo Hertz, para presupuestar capitales bajo riesgo, el modelo para presupuestar una compañía, y el modelo financiero de la SunOilCorporate.

- MODElos CORPORATIVOS. Tratan a la compañía de negocios como un sistema total. Representan un modelo para modelar el comportamiento de toda una corporación. Algunos modelos, son: el modelo de An- 
heuser - Busch, el de la IBM, el de Pillsburg, y Xerox.

\section{DISCUSIÓN}

Los procesos de simulación son, posiblemente, las herramientas más poderosas y populares para la toma de decisiones. Muchas empresas usan algún modelo de simulación en sus procesos. Algunas de las empresas han desarrollado modelos de simulación corporativos (para todos los niveles y funciones de la empresa); otras han instrumentado modelos de simulación para sus decisiones financieras, de mercadotecnia, producción o inventarios.

Un sistema puede ser representado por modelos matemáticos y lógicos, diseñados para tal fin. La complejidad de sistemas son simplificados por la técnica de simulación; por ejemplo, el comportamiento de sistemas económicos, sociales, administrativos, físicos, químicos, flujos, etc.

Esta técnica permite estudiar al sistema real sin deformarlo. Al simplificar el modelo, se consideran las condiciones que fundamentan la teoría del modelo en uso, pero de igual manera ocurre que muchas veces un modelo puede ser deformado, muy lejano al sistema real bajo estudio. Los procesos de simulación son herramientas muy efectivas de entrenamiento de personal y generan una visión completa del sistema bajo estudio, mucho más profunda y detallada que cualquier modelo analítico o numérico.

Es oportuno reconocer que los procesos de simulación no producen re- sultados óptimos, sino simplemente buenos. El tiempo de uso de computadora es elevado.

\section{DISEÑO DE EXPERIMENTOS DE SIMULACIÓN}

Cuando se ha validado el programa de computadora, se entra a la fase del diseño de experimentos que se requieren simular. Deben definirse las variables endógenas y exógenas y las estructuras funcionales que las relacionan.

Se eligen las distribuciones probabilísticas adecuadas a los parámetros aleatorios y se generan los números aleatorios que, de acuerdo a estas distribuciones, representan al sistema bajo estudio.

Esta fase está íntimamente ligada con el análisis de varianza de los resultados de la simulación, que mide el grado de asociación lineal entre variables endógenas y exógenas.

\section{GENERACIÓN DE NUMEROS ALEATORIOS}

Los números aleatorios se utilizan para introducir el comportamiento estocástico en el sistema bajo estudio. Estos números pueden encontrarse en tablas o generarse en una computadora. Para los fines de simulación manual, las tablas son más que suficientes. Si se trata de simular por computadora, las tablas son imprácticas y se requiere de proceso de autogeneración.

Los números generados en una computadora no son totalmente alea- 
torios, porque se obtienen de fórmulas preestablecidas, que cumplen con una serie de pruebas estadísticas de aleatoriedad. Por esta razón, a estos números se les denomina pseudoaleatorios.

\section{CONCLUSIONES}

Cuando la información es oportuna, relevante y confiable, se pueden mejorar y actualizar los modelos de simulación que se diseñan para un sistema dado.

En una línea de espera, se debe verificar si las llegadas de clientes o los servicios tienen una distribución dada. Para ello se requiere la realización de una serie de estadísticas para analizar si existen diferencias significativas.

A pesar de la gran versatilidad y amplia flexibilidad de la simulación, puede generar resultados imprecisos en el análisis de sistemas.
Los modelos a desarrollar pueden ser costosos y el procedimiento de validación del simulador puede ser difícil y tedioso.

El análisis de variancia es un conjunto de técnicas para analizar datos que se pueden usar para comprobar la hipótesis de que la media (o variancia), de una serie generada por un experimento de simulación en computadora es igual a la media (o variancia) de la serie observada correspondiente. La aplicación de esta técnica se basa en tres suposiciones importantes: normalidad, independencia estadística y una variancia común.

La prueba de Ji cuadrada, es la prueba estadística clásica que se puede emplear para comprobar la hipótesis de que el conjunto de datos generados por un modelo de simulación posee la misma distribución de frecuencia que el conjunto de datos históricos observados. 


\section{REFERENCIAS BIBLIOGRÁFICAS}

Ackoff, R. \& Sasieni, M. (1987). Fundamentos de Investigación de Operaciones. México: Ed. Limusa.

Antón, Howard (1984). Cálculo y Geometría Analitica. México: Ed. Limusa.

Apóstol, T. M. (1984). Introducción a la Teoría Analitica de Números. España: Ed. Reverté.

Bedworth, D. \& Bailey, J. (1988). Sistemas Integrados de Control de Producción, México: Ed. Limusa, Segunda Edic.

Buffa, E. (1977). Dirección de Operaciones Problemas y Modelos. México: Ed. Limusa, Segunda Edic.

Domínguez, J. (1995). Dirección de Operaciones. México: Ed. McGraw - Hill.

Hansen, D. \& Mowen, M. (1996). Administración de Costos. México: Ed. International.

Hiller, F. \& Lieberman, G. (1996). Introducción a la Investigación de Operaciones. México: Ed. McGraw - Hill, Cuarta Edic.

Mathur, K. \& Solow, D. (1996). Investigación de Operaciones, México: Ed Prentice - Hall Hispanoaméricana S.A., Segunda Edic.

Narasimhan, S.; Mcleavey, D. \& Billington, P. (1996). Planeación de la Producción y Control de Inventarios. México: Ed, Prentice Hall Hispanoamericana S.A., Tercera Edic.

Prawda, J. (1993). Métodos y Modelos de Investigación de Operaciones. México: Ed. Limusa Editores, Segunda Edic.

Shroeder, R. (1996). Administración de Operaciones. México: Ed. McGraw Hill, Tercera Edic. 